\title{
Téoros
}

Revue de recherche en tourisme

\section{Skiing à la française. Skier à la québécoise}

\section{Henri Jamet}

Volume 8, numéro 1, mars 1989

France-Québec

URI : https://id.erudit.org/iderudit/1080355ar

DOI : https://doi.org/10.7202/1080355ar

Aller au sommaire du numéro

Éditeur(s)

Université du Québec à Montréal

ISSN

0712-8657 (imprimé)

1923-2705 (numérique)

Découvrir la revue

Citer cet article

Jamet, H. (1989). Skiing à la française. Skier à la québécoise. Téoros, 8(1), 25-27. https://doi.org/10.7202/1080355ar d'utilisation que vous pouvez consulter en ligne.

https://apropos.erudit.org/fr/usagers/politique-dutilisation/ 
De la Laponic en passant par la Finlande, la Suède et la Norvège, le ski fut introduit en Suisse et en Allemagne en 1890. Son apparition en France fut un peu plus tardive. C'est aux bataillons de chasseurs alpins que revient le mérite de l'utilisation des skis en France. Ces militaires les utilisèrent pour la traversée de cols de montagne dans la région de Briançon dans les Alpes. Ce sont surtout le Club Alpin Français et le Touring Club de France qui contribuèrent à la diffusion de ce nouveau sport dans les hautes vallées des Alpes, des Pyrénées et du Jura, C'est en 1907 que fut tenu le premier concours international de ski à Montgenèvre dans le Briançonnais. Et puis c'est à Chamonix-Mont-Blanc en Haute-Savoie, en 1924, que furent tenus les premiers Jeux Olympiques d'hiver. Ils $n^{*}$ eurent pas grand-chose de commun avec les XVèmes Jeux de Galgary (1988). Le ski de descente fit de rapides progrès à partir de 1930 et il acquit ses lettres de noblesse en France grace aux victoires obtenues par le Megèvan Émile Allais, vainqueur au championnat du monde de la Fédération Internationale de Ski tenu à Chamonix et Megeve en 1937.

Le développement du ski et des sports d'hiver prirent une très forte expansion en France suite à la tenue des Xèmes Jeux Olympiques d'hiver à Grenoble en 1968, il $y$ a de cela 20 ans. La performance brillante des Jean-Claude Killy, triple médaillé d'or, Marielle Goitschel, Guy Perillat, ne fut pas étrangère au développement exceptionnel que le ski et les centres de ski allaient prendre par la suite.

\section{La grande époque du ski en France 1968-1988}

Avant les Jeux Olympiques de Grenoble de 1968, la France qui possédait des stations de ski de renommée internationale - comme Megeve, Chamonix - et connues - comme La Cluzaz ou Courchevel - n'arrivait pas à se classer au niveau de ses concurrents immédiats comme la Suisse et l'Autriche, au niveau des équipements parce que le ski y était moins pratiqué que chez ses voisins. Les Français attribuent en général aux Jeux Olympiques de Grenoble et aux 17 médailles glanees sur les neiges de Portillo au Chili en 1960 la fièvre du ski qui s'était cmparée de la France à cette époque.

C'est aussi le rapport de la Commission du tourisme pour le Veme Plan (1966-1970) qui prepara le climat en attirant l'attention sur la stagnation du ski en France et le grand bond en avant qui devait suivre. Ce Vème Plan faisait observer: Que les stations fran= caises n'wilisaient qu' 'une petite partie des beaux sites exploitables, ce qui n'était pas le cas dans d'autres pays concurrens. Les gisements de neige francais sont parmi les meilleurs d'Europe. Ils permettent de satisfaire autant les amateurs de sport que les amateurs de soleil sans craindre de nouveaux concurrents el doivent donc être considérés comme des richesses nationales à préserver puis à metre en valeur dans les meilleures conditions possibles.

C'est à cette époque que naquit le slogan officiel selon lequel la neige française pouvait devenir un "piège à devises" dans le cadre de la compétition internationale.

\section{Le plan neige 1971}

Le plan de 1971, élaboré pendant les années 1960 et mis en oeuvre entre 1971 et 1975 , allait marquer le paysage français de la montagne d'une façon indélébile.

Le plan prévoyait la construction de 150000 lits touristiques nouveaux en montagne et de 360000 lits entre 1970 et 1980 . Ce Plan Neige touchait vingt-trois anciennes stations telles que Font-Romeu, Serre-Chevalier, Montgenèvre, Les Gets, Morzine, Chamonix, Megève, Saint-Gervais, Les Contamines, La Cluzaz, etc... et vingt stations nouvelles dont l'aménagement avait déjà démarré en 1970 pour dix d'entre elles et dont dix autres ont été lanoées au cours du Vlème Plan entre 1971 et 1975.

Les stations du Plan Neige qui offraient 200000 lits en 1971 pouvaient en offrir 350000 cinq ans plus tard. La cadence annuelle de construction s'eleva à 30000 lits nouveaux par année, soit l'équivalent de 15000 chambres en occupation double, soit l'équivalent de 54 hôtels Québec/Hilton.

D'après les experts, l'expansion des nouvelles stations de ski ne s'est pas faite au détriment des stations anciennes. Le nombre de lits d'hébergement n'a pas cessé de s'accroître depuis ce temps sur une base annuelle de 30000 à 45000 lits. Chamonix disposait de 37080 lits, Megève de 36940 lits, Courchevel de 32435 lits, Morzine-Avoriaz de 22475 lits, Méribel de 22000 lits en 1987. 


\section{Accroissement du nombre de skieurs français}

S'ils n'étaient que quelques milliers au début de ce siècle, le nombre de skieurs français s'est aceru assez rapidement.

Nombre de skieurs français

\begin{tabular}{ll}
\hline 1960 (fin des années 60) & 1500000 \\
1972 & 2000000 \\
1980 & 5000000 \\
1988 & 7500000 \\
\hline
\end{tabular}

Parc des remontées mécaniques

\begin{tabular}{lr}
\hline 1960 & 400 appareils \\
1970 & 1809 appareils \\
1980 & 3270 appareils \\
1985 & 3596 appareils \\
1986 & 3672 appareils \\
1988 & 3800 appareils \\
\hline
\end{tabular}

Parmi les 3800 remontées mécaniques en exploitation, on dénombrait 2853 téléskis, 656 télésièges, 137 télécabines, 54 téléphèriques (dont le plus important à Courchevel détient le record du monde avec une cabine de 160 passagers) et quatre chemins de fer à crémaillère. Le plus récent de ces chemins de fer construit et mis en service en janvier 1988 à Val d'Isère peut transporter 3000 skieurs à l'heure sur une longueur de $2,3 \mathrm{~km}$ dont $1,6 \mathrm{~km}$ s'enfonce sous le rocher de Bellevarde.

Les départements de la Savoie et de la HauteSavoie regroupent à eux seuls plus de la moitié de ces installations (54\%), les Alpes du Sud $(17,6 \%)$, les Pyrénées (10\%), Isère + Drôme + Ardèche $(10,4 \%)$.

En 1987, plus de 130 millions de dollars canadiens ont été investis dans les seules remontées mécaniques. Le coüt de construction de ces appareils s'est élevé à 1,3 million canadien pour un télésiège de 300 mètres de dénivelée et d'un débit de 1500 skieurs à l'heure; à 4,2 millions de dollars canadiens pour un télécabine de 500 mètres et d'un debit de 1800 skieurs/heure. Le prix de certains téléphériques peut atteindre celui d'une rame de train TGV ou d'un Boeing 737. Au niveau de l'exploitation, les dépenses en personnel représentent $3,7 \%$ des coûts tandis que l'énergie électrique, $6 \%$.

\section{Le ski de fond en France}

Ce sport, quasi inexistant en France en 1950 , a connu une progression surprenante:

\begin{tabular}{lr}
\hline 1960 & 500 fondeurs \\
1970 & 10000 fondeurs \\
1978 & 600000 fondeurs \\
1980 & 1000000 fondeurs \\
\hline
\end{tabular}

Il y a maintenant en France 540 sites pour la pratique du ski de fond répartis dans 700 villages. Ils offrent $10000 \mathrm{~km}$ de pistes aménagées (balisées et damées) ainsi que 5000 km d'itinéraires moins aménagés. La Mecque du ski de fond se retrouve dans les Monts du Jura avec $32 \%$ des centres et les Alpes du Nord avec $29 \%$.

\section{Bilan des vingt dernières années}

Au cours des vingt dernières années, l'industrie des sports d'hiver dans les 144 stations de ski a généré plus d'une centaine de milliers de nouveaux emplois assurant dans des zones économiques fragiles la relève d'emplois agricoles et industriels en déclin. C'est le cas dans les Vallées de la Tarentaise en Savoie, de la Guisane dans les HautesAlpes. Aujourd'hui, il y a 10000 moniteurs de $\mathrm{ski}_{4}, 2000$ pisteurs secouristes, 13000 employés de remontées mécaniques, plusieurs dizaines de milliers d'employés dans le secteur de l'hôtellerie, de la restauration, des transports et des services.

Après vingt ans de rattrapage, l'industrie française des sports d'hiver rivalise maintenant avec ses voisins Autrichiens, Suisses, Italiens. On y dénombre 7500000 de skieurs alpins en 1988 et 3800 remontées mécaniques. Elle a généré une industrie de pointe:

Rossignol: no. 1 mondial (ski);

Salomon: no. 1 mondial (fixations, bottes de ski); Pomagalski: no. 1 mondial (remontées
mécaniques).

La mise en oeuvre d'infrastructures lourdes a nécessité des immobilisations importantes. Chaque lit créé représente un investissement de 20000 à 30000 dollars canadiens. Après cet effort exceptionnel, la machine commence à manifester des signes d'essoufflement. Pour la première fois en 1984-1985, les taux de départs en vacances aux sports d'hiver ont baissé d'une façon significative; il y a eu une autre légère baisse en 1985-1986 puis une reprise par la suite. Pour l'hiver 1987-1988, les taux de départs représentaient $35,5 \%$ de ceux qui partaient pour un total de 5 millions de touristes. En moyenne, les séjours aux sports d'hiver durent huit à neuf jours et s'adressent massivement à la categorie des 20 aे 49 ans $(45,6 \%)$. Les 14 à 19 ans représentent $14 \%$ et les 50 ans et plus: $18,4 \%$ des partants.

Les Alpes du Nord sont les premières montagnes recherchées par les skieurs; viennent ensuite les Alpes du Sud qui tendent même à les rattraper. En 1985, les premières accueillaient 3168000 séjours et en 1986: 2947000 séjours et les dernières: 748000 séjours en 1985 et 1181000 en 1986. Suivent les Pyrénées puis le Jura (environ 300000 sejours), le Massif Central (200 000 sćjours) et les Vosges (120 000 séjours); les trois perdent du terrain.

Les skieurs français vont pratiquer aussi leur sport en Suisse (137 000 sćjours en 1986). en Autriche (82 000 séjours) et en Espagne (55 000 séjours). D'après un rapport de la Délégation à l'Aménagement du Territoire et à l'Action Regionale:

"L'hypothèse la plus plausible pour les années qui viennent est que le marché français ne connaitra plus les croissances exceptionnelles des dernières décennies... La France devra dans le cadre de la compétition trouver ailleurs des marchés qui ne sont pas encore trop sollicités tels que la Scandinavie, l'Angleterre, l'Espagne et les marchés nord-américains",

\section{Historique du ski à la québécoise}

La première apparition du ski remonte au Québec en 1879 lorsqu'un immigrant norvégien fit le trajet de Montréal a Québec sur "des chaussures de ski norvégiennes". La pratique de l'activité débuta en 1890 . Le Montréal Ski Club fut mis sur pied en 1904 , le Club de ski de Québec en 1908 et celui de Trois-Rivières en 1911.

Le premier remonte-pente fut introduit au Québec en 1929 à Foster Hill à cốté de Shawbridge dans les Laurentides (il s'agissait en fait $d^{+} u n$ cabble sans fin très rudimentaire actionné par un moteur V8). Le train de neige (le petit train du Nord) débuta ses opérations en 1927.

C'est surtout la tenue des Jeux d'hiver à Lake Placid dans l'État de New-York en 1932 qui lançal le ski sur le continent nord-américain. Au Canada, le nombre de stations de ski passa de 53 à 119 entre 1961 et 1965 et le nombre de remonte-pentes de 90 à 235 ; de 1965 a 1976, le nombre de stations de ski passa de 119 à 206 et le nombre de remontepentes de 235 à 731 .

Il y avait au Québec, au cours de l'hiver 1985-1986, 106 centres de ski alpin. Le parc était constitué par 9 centres majeurs, 12 centres intermédiaires présentant plus de 250 mètres de dénivelé et plus de 100 lits à la base. Il y avait aussi 25 centres intermédiaires de loisirs présentant moins de 250 mètres de dénivelée et moins de 100 lits à la base et, enfin, 60 petits centres.

La dénivelée des centres québécois varie en moyenne de 131 mètres dans les petits centres à 452 mètres dans les centres majeurs. La dénivelée du Mont Tremblant est de 656 mètres. Ce sont surtout les 9 centres majeurs qui peuvent se comparer avec les gros centres de ski de la Nouvelle-Angleterre. Contrairement à la situation d'équilibre observée aux USA et en France, le ski alpin au Québec connait une croissance importante depuis 5 ans. La clientèle québécoise des skieurs a pratiquement doublé entre 1982 et 1987 passant de $7,5 \%$ à $15 \%$; le nombre de skieurs québécois âgés de 18 ans et plus est passé de 661000 en 1984-1985 à 850000 en 1985-1986, soit une augmentation de $28 \%$. Il y aurait actuellement près de 1 million de skieurs actifs. La fréquentation des centres 
de ski représente environ 10 millions de jours/skieurs dont $90 \%$ sont générés par la clientèle québécoise et $10 \%$ par la clientèle americaine et des autres provinces canadiennes, principalement de $\mathrm{I}^{\dagger}$ Ontario.

\section{Impact économique}

L'industrie du ski a généré des dépenses de 425 millions de dollars pendant l'hiver 1986-1987; 200 millions ont été dépensés dans les centres de ski proprement dits; 190 millions au niveau de l'hôtellerie et de la restauration et 75 millions au niveau de l'achat d'équipements et d'articles de sport. L'industrie a généré des recettes fiscales de 62 millions au niveau de la province et de 33 millions au niveau fédéral, soit un total de 95 millions.

Le chiffre d'affaires s'est élevé en moyenne de 160000 dollars pour les petits centres à 1 million pour les centres intermédiaires de loisirs, 4,4 millions pour les centres intermédiaires touristiques et 6,4 millions pour les centres majeurs.

La vente de billets ne représente que $56 \%$ du revenu d'un centre de ski, les autres activités telles que boutiques, écoles de ski, $14 \%$ et l'hébergement et la restauration, $40 \%$. Enfin, 10450 emplois par année sont liés directement ou indirectement à l'activité du ski alpin dont 3750 dans les stations de ski.

\section{L'entente auxiliaire Canada-Québec}

Au cours de la période de 1983 à 1986, 56 centres de ski ont bénéficié de ce programme auquel se sont greffees d'autres formes de soutien. Le coup de pouce du secteur public de 70 millions de dollars a généré des investissements de l'ordre de 180 millions: 75 millions sont allés à la modernisation et à l'expansion des équipements de montagne; 55 millions ont été utilisés à l'hébergement et aux services au bas des pentes; 30 millions ont été consacrés à l'amélioration des infrastructures et des services collectifs; 20 millions ont été utilisés pour l'amélioration générale des centres ( 46 centres fabriquent de la neige artificielle et 61 ont des pistes eclairées le soir).

Le développement de l'hébergement au pied des pentes est bien amorce. Depuis deux ans environ, des projets réalisés ou en voie de réalisation totalisent 100 à 125 millions de dollars. D'aptès une étude récente réaliséc par la firme CEGIR "la transformation des principaux centres de ski, une dizaine au maximum, en véritables póles touristiques quatre-saisons, passe par l'emergence d'une fonction de commercialisation beaucoup plus poussée qu'elle ne l'est à l'heure actuelle'" et également par la consolidation des activités d'après-ski qui font la notoriété, la prospérité de certaines grandes stations européennes ou américaines. La neige est indispensable dans les centres de ski, qu'elle soit naturelle ou artificielle mais il faut plus que de la neige pour attirer et conserver la clientèle. Il faut offrir des activités culturelles et des compétitions sportives d'envergure.

\section{Portrait-type des grandes stations québécoises}

Le Mont Tremblant, avec ses 48 ans de service, fait figure de précurseur: ses 14 hôtels, ses 41 pistes balisées, ses 10 remontées mécaniques en font une station de calibre international. Le Mont Sainte-Anne inauguré en 1966 avec sa dénivelée de 625 mètres, ses 14 remontées mécaniques, ses 38 pistes, sa proximité de Québec, est devenu une destination de ski internationale. Le Mont Sainte= Anne s'est doté d'un nouvel hôtel de 89 chambres, on a construit au pied des pentes un centre de villégiature avec boutiques et, récemment, un complexe sportif. Au cours de l'été, près de 7 millions ont été investis au Mont Sainte-Anne dont 600000 dollars pour améliorer le domaine skiable.

Le massif de la Petite Rivière Saint-François comporte actuellement une dizaine de pistes aménagées mais aucune remontée mécanique. Les descentes s"effectuent par petits groupes sous la conduite d'un guide et les remontées s'effectuent en autobus. Ce centre de ski international en devenir, parce qu'il possède la plus haute dénivelée du Québec, doit éventuellement faire l'objet de travaux évalués à 100 millions de dollars pour la première phase; le massif, qui est jumelé avec la station les Arcs, profitera de l'expertise et de la renommée de cette dernière.

Le programme d'investissements publics et privés amorcé au Québec en 1983 a permis à l'industrie québécoise des sports d'hiver de reprendre une place enviable de la méme façon que le Plan Neige mis en oeuvre par les Français a permis à la France de rattraper son retard par rapport à ses voisins Suisses et Autrichiens. Il n'en demeure pas moins que, pour conserver une position concurrentielle, la Belle Province et la France devront continuer à être imaginatives, constantes et agressives au niveau du développement, de l'évolution du produit et du marketing.

\section{Les enjeux du futur}

Les vacanciers des sports d'hiver ont deja adopté les règles du libre-echange depuis quelques hivers. A l'heure actuelle, l'offre est considérable, les compétiteurs des Québécois et des Français sont légion. La concurrence est féroce et l'offre s'est raffinee. Le retour à la station-village s'accentue et la reconquête du village ancestral refait surface. Les marchands des blocs-appartements des neiges doivent se faire plus discrets. Le style montagnard qui s"harmonise avec son environnement fait recette partout où on l'avait foulé aux skis.

Les stations toutes neuves d'Amérique suivent cette tendance: c'est l'Europe servie a l'américaine. L'urbanisme s'inspire des stations de ski à l'européenne où l'on tend à évacuer les voitures et où tout est accessible en peu de temps à pied ou en ski. Pour consoli- der sa position concurrentielle, le Québec devra accentuer sa priorité sur l'hébergement au pied des pentes comme l'a fait le groupe des "91 majeures françaises" qui possède 1000000 de lits touristiques, soit 11000 lits (5 000 chambres) en moyenne par station. Le Québec est encore loin du compte à l'intérieur de ses "9 majeures" mais il ne devrait pas être réticent à se donner sur les marchés intérieurs et extérieurs une véritable image de pays de sports d'hiver comme les concurrents européens ont si bien réussi à le faire. Pour cela le Québec aurait avantage à améliorer son réseau de transport terrestre et surtout aérien.

La France, de son côté, devra continuer son effort pour corriger les exces de certains grands constructeurs qui ont défiguré certains villages et sites de montagne.

Une collaboration plus intense entre les stations de ski françaises et québécoises devrait s'accentuer pour affronter la competition. Le Québec avec son slogan "skiing à la française" a fait un premier petit pas. C'est au tour de la France de skier à la québécoise!

Note
(1) Ce slogan publicitaire fut utlise par le Service de mar-
keting de Tourisme Ouebec pour vendre la neige que-
bécoise sur le marché américain 